\title{
湿法合成 $\mathrm{LiNi}_{x} \mathrm{Co}_{y} \mathrm{Mn}_{1-x-y} \mathrm{O}_{2}$ 的 $E$-pH 图绘制及其电性能
}

\author{
李 玲 ${ }^{1,2}$, 李运姣 ${ }^{1,2}$, 徐 斌 $^{3}$ 卢伟胜 ${ }^{2}$, 苏千叶 ${ }^{1,2}$, 陈永祥 ${ }^{1,2}$, 李 林 $^{1}$
}

(1. 中南大学 冶金与环境学院, 长沙 410083; 2. 中信大锰矿业有限责任公司, 南宁 530028; 3. 青海快驴高新技术 有限公司, 西宁 810000)

摘 要: 通过热力学计算获得了 $\mathrm{Li}-\mathrm{Ni}-\mathrm{Co}-\mathrm{Mn}-\mathrm{H}_{2} \mathrm{O}$ 系中各物质的热力学数据, 从而绘制了 $25^{\circ} \mathrm{C}$ 和 $200^{\circ} \mathrm{C}$ 、离子活度 为 1.00 下的 $\mathrm{Li}-\mathrm{Ni}-\mathrm{Co}-\mathrm{Mn}-\mathrm{H}_{2} \mathrm{O}$ 系电位-pH 图。热力学分析结果表明: 温度为 $25^{\circ} \mathrm{C}$ 时, 在 $\mathrm{pH} 3 \sim 13$ 范围内, 水溶液中 未出现 $\mathrm{LiNi}_{x} \mathrm{Co}_{y} \mathrm{Mn}_{1-x-y} \mathrm{O}_{2}$ 稳定区域; 随着温度升高, $\mathrm{Li}-\mathrm{Ni}-\mathrm{Co}-\mathrm{Mn}-\mathrm{H}_{2} \mathrm{O}$ 系中各物质的稳定区域向低 $\mathrm{pH}$ 和低电位方向 移动。在温度为 $200^{\circ} \mathrm{C}, \mathrm{pH}$ 为 9.7 13.0 的水溶液中出现了 $\mathrm{LiNi}_{x} \mathrm{Co}_{y} \mathrm{Mn}_{1-x-y} \mathrm{O}_{2}$ 稳定区域。这说明在一定温度下, 水溶液 中合成 $\mathrm{LiNi}_{x} \mathrm{Co}_{y} \mathrm{Mn}_{1-x-y} \mathrm{O}_{2}$ 是可能的, 且提高温度有利于合成反应进行。进一步通过实验验证, 以 $\left(\mathrm{Ni}_{0.5} \mathrm{Co}_{0.2} \mathrm{Mn}_{0.3}\right)(\mathrm{OH})_{2}$ 前驱体和 $\mathrm{LiOH} \cdot \mathrm{H}_{2} \mathrm{O}$ 为原料, 在水溶液中成功获得具有 $\alpha-\mathrm{NaFeO}_{2}$ 层状结构的锂镍钴锰四元前驱体, 经过热处理后 得到循环稳定性良好的 $\mathrm{LiNi}_{0.5} \mathrm{Co}_{0.2} \mathrm{Mn}_{0.3} \mathrm{O}_{2}$ 正极材料。实验结果证明, 所绘制 Li-Ni-Co-Mn- $\mathrm{H}_{2} \mathrm{O}$ 系 E-pH 图是可靠 的, 且湿法合成 $\mathrm{LiNi}_{0.5} \mathrm{Co}_{0.2} \mathrm{Mn}_{0.3} \mathrm{O}_{2}$ 正极材料具有良好的循环性能。

关 键 词: E-pH 图; Li-Ni-Co-Mn- $\mathrm{H}_{2} \mathrm{O}$ 系; 锂离子电池; 正极材料

中图分类号: TM912 文献标识码: A

\section{$\mathrm{LiNi}_{x} \mathrm{Co}_{y} \mathrm{Mn}_{1-x-y} \mathrm{O}_{2}$ Cathode Material Synthesized through Construction of $E$-pH Diagram and Its Electrochemical Performance}

\author{
LI Ling ${ }^{1,2}$, LI Yun-Jiao ${ }^{1,2}$, XU Bin ${ }^{3}$, LU Wei-Sheng ${ }^{2}$, SU Qian-Ye ${ }^{1,2}$, CHEN Yong-Xiang ${ }^{1,2}$, LI Lin ${ }^{1}$ \\ (1.School of Metallurgy and Environment, Central South University, Changsha 410083, China;2. Citic Dameng Mining Indus- \\ tries Limited, Nanning 530028, China;3. Qinghai Quick Donkey Hi Tech Co., Ltd., Xining 810000, China)
}

\begin{abstract}
The thermodynamic data of various species in $\mathrm{Li}-\mathrm{Ni}-\mathrm{Co}-\mathrm{Mn}-\mathrm{H}_{2} \mathrm{O}$ system is obtained by thermodynamics calculation, and $\mathrm{E}-\mathrm{pH}$ diagrams for Li-Ni-Co-Mn- $\mathrm{H}_{2} \mathrm{O}$ system with activity 1.00 at $25^{\circ} \mathrm{C}$ and $200{ }^{\circ} \mathrm{C}$ were constructed. From $E-p H$ diagrams, it shows that there is no predominant region of the $\mathrm{LiNi}_{x} \mathrm{Co}_{y} \mathrm{Mn}_{1-x-y} \mathrm{O}_{2}$ composite oxide in $\mathrm{pH}$ range of $3 \sim 13$ at $25^{\circ} \mathrm{C}$. However, the stability region of various species expands towards the low $\mathrm{pH}$ and low potential zones as the temperature increases. When $\mathrm{pH}$ is between $9.7 \sim 13.0$ at $200^{\circ} \mathrm{C}$, synthesis of $\mathrm{LiNi}_{x-}$ $\mathrm{Co}_{y} \mathrm{Mn}_{1-x-y} \mathrm{O}_{2}$ via aqueous process is thermodynamically possible, and high temperature is favorable. Experimental results showed that the composite precursor (LNCM) with $\alpha-\mathrm{NaFeO}_{2}$ structure was successfully prepared in aqueous solution by using $\left(\mathrm{Ni}_{0.5} \mathrm{Co}_{0.2} \mathrm{Mn}_{0.3}\right)(\mathrm{OH})_{2}$ as precursor and $\mathrm{LiOH} \cdot \mathrm{H}_{2} \mathrm{O}$ as raw material. $\mathrm{LiNi}_{0.5} \mathrm{Co}_{0.2} \mathrm{Mn}_{0.3} \mathrm{O}_{2}$ cathode materials were then obtained by post heat treatment and following tests exhibited excellent cycling performance. These experimental results are in consistent with the information given in $E$-pH diagram of the Li-Ni-Co-Mn- $\mathrm{H}_{2} \mathrm{O}$ system, and the as-prepared $\mathrm{LiNi}_{0.5} \mathrm{Co}_{0.2} \mathrm{Mn}_{0.3} \mathrm{O}_{2}$ cathode materials show excellent cycling performance.
\end{abstract}

Key words: $E$-pH diagram; $\mathrm{Li}-\mathrm{Ni}-\mathrm{Co}-\mathrm{Mn}-\mathrm{H}_{2} \mathrm{O}$ system; lithium ion battery; cathode material

收稿日期: 2017-04-20; 收到修改稿日期：2017-09-06

基金项目：广西八桂学者计划(2011A025); 广西崇左市重大科技专项([2017]11)

Glorious Laurel Scholar Program(2011A025); The major Science \& Technology ([2017]11)

作者简介: 李 玲(1989-), 女, 硕士. E-mail: 928012218@qq.com

通讯作者: 李运姣, 教授. E-mail: yunjiao_li@csu.edu.cn 
$E-\mathrm{pH}$ 图可以表示反应体系中化学或电化学反 应的平衡条件和体系中各组分稳定区域等, 它广 泛应用于腐蚀化学、湿法冶金和电化学等领域，并 可应用于材料领域指导复杂化合物的湿化学合成, 如 $\mathrm{Li}_{2} \mathrm{TiO}_{3}{ }^{[1]} 、 \mathrm{LiCoO}_{2}{ }^{[2]}$ 等。已有报道的 $\mathrm{Li}-\mathrm{Me}-\mathrm{H}_{2} \mathrm{O}$ 系 $E-\mathrm{pH}$ 图主要局限于单一过渡金属体系, 如 Li-Ni- $\mathrm{H}_{2} \mathrm{O}^{[3]}$ 系、 Li-Co- $\mathrm{H}_{2} \mathrm{O}^{[4]}$ 系、 Li-Mn- $\mathrm{H}_{2} \mathrm{O}^{[5]}$ 系和 $\mathrm{Li}-\mathrm{Ti}-\mathrm{H}_{2} \mathrm{O}^{[1,6]}$ 系等, 且多局限于常温水溶液体系。对 于多种过渡金属复杂体系在高温下的 $E-\mathrm{pH}$ 图, 目 前还未见有报道。

涉及多种过渡金属的 $\mathrm{LiNi}_{x} \mathrm{Co}_{y} \mathrm{Mn}_{1-x-y} \mathrm{O}_{2}$ (其中 $x+y<1, x>0$, 且 $y>0$ )正极材料通过协同效应, 综合了 $\mathrm{LiCoO}_{2}, \mathrm{LiNiO}_{2}$ 和 $\mathrm{LiMnO}_{2}$ 三种材料的特点, 具有放 电比容量高、循环稳定性良好、成本低和安全性好 等优点, 是最具发展潜力的锂离子电池正极材料之 - ${ }^{[7-8]}$ 。目前, 合成 $\mathrm{LiNi}_{x} \mathrm{Co}_{y} \mathrm{Mn}_{1-x-y} \mathrm{O}_{2}$ 材料的方法主 要有高温固相法 ${ }^{[9]}$ 、共沉淀法 ${ }^{[10]}$ 、溶胶凝胶 ${ }^{[11]}$ 和流变 相反应法 ${ }^{[12]}$ 等。合成方法会直接影响所得材料的结 晶度、相纯度、颗粒形貌和颗粒粒度等物理特性, 进 而影响材料的电化学性能 ${ }^{[13-14]}$ 。许多研究者尝试新的 合成方法来提高三元材料的电化学性能。Seung-Taek 等 ${ }^{[15]}$ 以共沉淀法所得球形 $\left[\mathrm{Ni}_{1 / 3} \mathrm{Co}_{1 / 3} \mathrm{Mn}_{1 / 3}\right](\mathrm{OH})_{2}$ 前 驱体和 $\mathrm{LiOH}$ 为原料, 在 $170^{\circ} \mathrm{C}$ 高压釜内反应 $4 \mathrm{~d}$ 后, 再经过 $800^{\circ} \mathrm{C}$ 热处理 $5 \mathrm{~h}$, 得到 $\mathrm{Li}\left[\mathrm{Ni}_{1 / 3} \mathrm{Co}_{1 / 3} \mathrm{Mn}_{1 / 3}\right] \mathrm{O}_{2}$ 正极材料, 其在 $0.2 C 、 2.8 \sim 4.3 \mathrm{~V}$ 电压范围放电比容 量为 $157 \mathrm{mAh} / \mathrm{g}$ 。由于缺乏相应的 Li-Ni-Co-Mn- $\mathrm{H}_{2} \mathrm{O}$ 系 $E-\mathrm{pH}$ 图作指导, 人们在尝试水溶液法合成 $\mathrm{LiNi}_{x} \mathrm{Co}_{y} \mathrm{Mn}_{1-x-y} \mathrm{O}_{2}$ 材料的过程中往往具有较大的盲 目性。

本工作在查询相关文献和热力学数据的基础上, 通过有关热力学计算, 绘制了 Li-Ni-Co-Mn- $\mathrm{H}_{2} \mathrm{O}$ 系 在 $25^{\circ} \mathrm{C}$ 和 $200^{\circ} \mathrm{C}$ 、离子活度为 1.00 下的 $E-p H$ 图, 从 热力学角度分析了在水溶液中合成 $\mathrm{LiNi}_{x} \mathrm{Co}_{y} \mathrm{Mn}_{1-\mathrm{x}-\mathrm{y}} \mathrm{O}_{2}$ 材料的可行性。并通过验证实验, 在水溶液中成功 合成了 $\mathrm{LiNi}_{0.5} \mathrm{Co}_{0.2} \mathrm{Mn}_{0.3} \mathrm{O}_{2}$ 正极材料。

\section{Li-Ni-Co-Mn- $\mathrm{H}_{2} \mathrm{O}$ 系 E-pH 图绘制}

根据 $\mathrm{Li} 、 \mathrm{Ni} 、 \mathrm{Co} 、 \mathrm{Mn}$ 在水溶液中的行为及其 相互作用原理，吉布斯自由能可表示为:

$$
\Delta_{\mathrm{f}} G_{\mathrm{T}}^{\varnothing}=\Delta_{\mathrm{f}} G_{298.15}^{\varnothing}-(T-298.15) \Delta S_{298.15}^{\varnothing}
$$

式中 $\Delta_{\mathrm{r}} G_{T}^{\varnothing} 、 \Delta_{\mathrm{r}} G_{298.15}^{\varnothing}$ 表示一定温度 $T$ 和 $298.15 \mathrm{~K}$ 下 的标准吉布斯自由能变化, $\mathrm{kJ} / \mathrm{mol} ; T$-温度, $\mathrm{K}$; $\Delta S_{298}^{\varnothing}$ 绝对熵值变化。通过计算得到各反应式在不同 条件下的反应吉布斯自由能, 反应吉布斯自由能分
别与 $E$ 和 $\mathrm{pH}$ 关系如下:

$$
\begin{gathered}
\varphi_{T}=-\frac{\Delta_{\mathrm{r}} G_{T}^{\varnothing}}{z \mathrm{~F}}-\frac{2.303 n R T}{z \mathrm{~F}} \mathrm{pH}-\frac{2.303 \mathrm{R} T}{z \mathrm{~F}} \lg \frac{a_{\mathrm{B}}^{\mathrm{b}}}{a_{\mathrm{A}}^{\mathrm{a}}} \\
\mathrm{pH}_{T}=-\frac{\Delta_{\mathrm{r}} G_{T}^{\varnothing}}{2.303 n \mathrm{R} T}-\frac{\mathrm{b}}{n} \lg a_{\mathrm{B}}+\frac{\mathrm{a}}{n} \lg a_{\mathrm{A}}
\end{gathered}
$$

式中 $\varphi_{T}$ 一反应在一定条件下的电极电位, $\mathrm{V} ; \mathrm{z}^{-}$电子 迁移数; $\mathrm{F}$ 一法拉第常数; $\mathrm{R}$-普适气体常数, $\mathrm{J} /(\mathrm{mol} \cdot \mathrm{K})$; $n$ 一参加反应的 $\mathrm{H}^{+}$数; $a_{\mathrm{H}_{2} \mathrm{O}} 、 a_{\mathrm{B}} 、 a_{\mathrm{A}}$ 和 $a_{\mathrm{H}^{+}}-\mathrm{H}_{2} \mathrm{O}$ 、

B、 $\mathrm{A} 、 \mathrm{H}^{+}$的活度。

从上述公式可以看出，为了得到各反应在不同 温度下电势 $E 、 \mathrm{pH}$ 和离子活度间的关系, 需要获得 体系中各物质的标准吉布斯自由能和标准熵值。

Li-Ni-Co-Mn- $\mathrm{H}_{2} \mathrm{O}$ 系中各物质的标准吉布斯自由能 可以通过查询热力学手册 ${ }^{[16]}$ 和相关文献 ${ }^{[3-5]}$ 获得; 各物质在标准状态下的标准熵值除通过查询热力学 手册 ${ }^{[16]}$ 和相关文献 ${ }^{[3-5]}$ 获得以外, 还可采用各种经 验公式 ${ }^{[1]}$, 如氧化物商值加合法、Connick 公式 ${ }^{[17]}$ 和阳离子熵值与按阳离子平均价数计算所得阴离子 熵值之和法等。

根据计算获得 Li-Ni-Co-Mn- $\mathrm{H}_{2} \mathrm{O}$ 系中各反应在 不同温度下电势 $E 、 \mathrm{pH}$ 和离子活度间的关系, 从而 绘制了 $\mathrm{Li}-\mathrm{Ni}-\mathrm{Co}-\mathrm{Mn}-\mathrm{H}_{2} \mathrm{O}$ 系在 $25^{\circ} \mathrm{C} 、 200^{\circ} \mathrm{C}$, 离子 活度为 1.00 下的 $E-\mathrm{pH}$ 图, 如图 1 和图 2 所示。

图中垂直线阴影部分表示 $\mathrm{LiNiO}_{2} 、 \mathrm{LiCoO}_{2}$ 和 $\mathrm{LiMnO}_{2}$ 在 Li-Ni-Co-Mn- $\mathrm{H}_{2} \mathrm{O}$ 系 E-pH 图中的共同区 域, 即 $\mathrm{LiNi}_{x} \mathrm{Co}_{y} \mathrm{Mn}_{1-x-y} \mathrm{O}_{2}$ 稳定区域; 而斜线阴影部 分表示 $\mathrm{Ni}(\mathrm{OH})_{2} 、 \mathrm{Co}(\mathrm{OH})_{2}$ 和 $\mathrm{Mn}(\mathrm{OH})_{2}$ 共同区域，即 $\left(\mathrm{Ni}_{x} \mathrm{Co}_{y} \mathrm{Mn}_{1-\mathrm{x}-\mathrm{y}}\right)(\mathrm{OH})_{2}$ 稳定区域。图 1 是 Li-Ni-Co-Mn$\mathrm{H}_{2} \mathrm{O}$ 系在 $25^{\circ} \mathrm{C}$ 下的 $E-\mathrm{pH}$ 图, 从图中可以看出, 左边 存在一个较大的 $\mathrm{Ni}^{2+} 、 \mathrm{Co}^{2+}$ 和 $\mathrm{Mn}^{2+}$ 共同稳定区域, 右 中部存在一个类似长方形的 $\left(\mathrm{Ni}_{x} \mathrm{Co}_{y} \mathrm{Mn}_{1-x-y}\right)(\mathrm{OH})_{2}$ 共

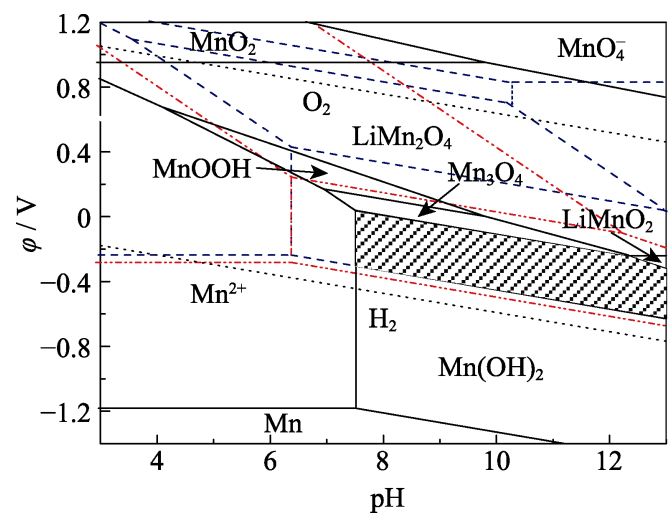

图 1 Li-Ni-Co-Mn- $\mathrm{H}_{2} \mathrm{O}$ 系在 $25^{\circ} \mathrm{C}$ 下的 $E-\mathrm{pH}$ 图

Fig. 1 Potential-pH diagrams of Li-Ni-Co-Mn- $\mathrm{H}_{2} \mathrm{O}$ system at $25^{\circ} \mathrm{C}$

( - - - - Li-Ni- $\mathrm{H}_{2} \mathrm{O} ;$---- Li-Co- $\left.\mathrm{H}_{2} \mathrm{O} ;-\mathrm{Li}-\mathrm{Mn}-\mathrm{H}_{2} \mathrm{O}\right)$ 


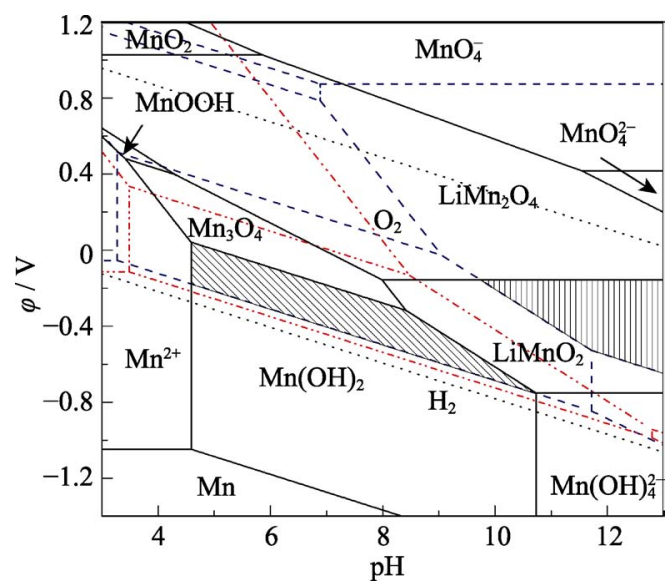

图 $2 \mathrm{Li}-\mathrm{Ni}-\mathrm{Co}-\mathrm{Mn}-\mathrm{H}_{2} \mathrm{O}$ 系在 $200^{\circ} \mathrm{C}$ 下的 $E-\mathrm{pH}$ 图

Fig. 2 Potential-pH diagrams of Li-Ni-Co-Mn- $\mathrm{H}_{2} \mathrm{O}$ system at $200^{\circ} \mathrm{C}$

( - - - Li-Ni- $\mathrm{H}_{2} \mathrm{O} ;$ - - - Li-Co- $\left.\mathrm{H}_{2} \mathrm{O} ;-\mathrm{Li}-\mathrm{Mn}-\mathrm{H}_{2} \mathrm{O}\right)$

沉淀区域。两个共同稳定区域相交于 $\mathrm{pH} 7.5$ 处，表明 当溶液的 $\mathrm{pH}$ 高于 7.5 时, 金属离子 $\mathrm{Ni}^{2+} 、 \mathrm{Co}^{2+}$ 和 $\mathrm{Mn}^{2+}$ 可水解成复合氢氧化物 $\left(\mathrm{Ni}_{x} \mathrm{Co}_{y} \mathrm{Mn}_{1-x-y}\right)(\mathrm{OH})_{2}$ 共沉淀。 在 $25^{\circ} \mathrm{C}$ 下, $\mathrm{Li}-\mathrm{Ni}-\mathrm{Co}-\mathrm{Mn}-\mathrm{H}_{2} \mathrm{O}$ 系 $\mathrm{E}-\mathrm{pH}$ 图右中部的边 缘处, 存在一小三角形的 $\mathrm{LiMnO}_{2}$ 稳定区域, 它位 于 $\mathrm{LiNiO}_{2}$ 和 $\mathrm{LiCoO}_{2}$ 稳定区域之外。因此, 该温度下 图中所示的 $\mathrm{pH}$ 和电位范围内不存在 $\mathrm{LiNiO}_{2} 、 \mathrm{LiCoO}_{2}$ 和 $\mathrm{LiMnO}_{2}$ 共同稳定区域, 即 $\mathrm{LiNi}_{x} \mathrm{Co}_{y} \mathrm{Mn}_{1-x-y} \mathrm{O}_{2}$ 共 同稳定区域, 说明该温度下难以在水溶液中合成 $\mathrm{LiNi}_{x} \mathrm{Co}_{y} \mathrm{Mn}_{1-x-y} \mathrm{O}_{2}$ 材料。

图 2 是 $\mathrm{Li}-\mathrm{Ni}-\mathrm{Co}-\mathrm{Mn}-\mathrm{H}_{2} \mathrm{O}$ 系在 $200^{\circ} \mathrm{C}$ 下的 $E-\mathrm{pH}$ 图, 从图中可以看出, $E-\mathrm{pH}$ 图中存在 $\left(\mathrm{Ni}_{x} \mathrm{Co}_{y} \mathrm{Mn}_{1-x-y}\right)$ $(\mathrm{OH})_{2}$ 和 $\mathrm{LiNi}_{x} \mathrm{Co}_{y} \mathrm{Mn}_{1-x-y} \mathrm{O}_{2}$ 稳定区域, 即 $200{ }^{\circ} \mathrm{C}$ 下 $\left(\mathrm{Ni}_{x} \mathrm{Co}_{y} \mathrm{Mn}_{1-x-y}\right)(\mathrm{OH})_{2}$ 和 $\mathrm{LiNi}_{x} \mathrm{Co}_{y} \mathrm{Mn}_{1-x-y} \mathrm{O}_{2}$ 均可稳定 存在与水溶液中, 这说明在高温条件下通过水溶 液法制备 $\mathrm{LiNi}_{x} \mathrm{Co}_{y} \mathrm{Mn}_{1-x-y} \mathrm{O}_{2}$ 材料在热力学上是可 能的。 $\mathrm{LiNi}_{x} \mathrm{Co}_{y} \mathrm{Mn}_{1-x-y} \mathrm{O}_{2}$ 稳定区域主要由 $\mathrm{LiNiO}_{2}$ 和 $\mathrm{LiMnO}_{2}$ 稳定区域决定, 因为 $\mathrm{LiCoO}_{2}$ 稳定区域几 乎完全包含了 $\mathrm{LiNiO}_{2}$ 和 $\mathrm{LiMnO}_{2}$ 稳定区域, $\mathrm{LiNi}_{x} \mathrm{Co}_{y} \mathrm{Mn}_{1-x-y} \mathrm{O}_{2}$ 共同稳定区域的 $\mathrm{pH}$ 范围大约为 9.7 13.0。从图中还可以看出随着 $\mathrm{pH}$ 的增加, 溶液 中的金属离子 $\mathrm{Ni}^{2+} 、 \mathrm{Co}^{2+}$ 和 $\mathrm{Mn}^{2+}$ 水解成复合氢氧 化物 $\left(\mathrm{Ni}_{x} \mathrm{Co}_{y} \mathrm{Mn}_{1-x-y}\right)(\mathrm{OH})_{2}$, 在溶液中存在 $\mathrm{Li}^{+}$和 $\mathrm{pH}$ 值进一步增加的条件下可转化为嵌锂复合氧化物 $\mathrm{LiNi}_{x} \mathrm{Co}_{y} \mathrm{Mn}_{1-x-y} \mathrm{O}_{2}$ 。

与图 1 相比, 图 2 中 $\left(\mathrm{Ni}_{x} \mathrm{Co}_{y} \mathrm{Mn}_{1-x-y}\right)(\mathrm{OH})_{2}$ 共沉淀 区往低 $\mathrm{pH}$ 方向移动、电势范围缩小, 区域变得更加 狭长。当温度由 $25^{\circ} \mathrm{C}$ 升高到 $200^{\circ} \mathrm{C}$ 时, $\mathrm{Ni}^{2+} 、 \mathrm{Co}^{2+}$ 和 $\mathrm{Mn}^{2+}$ 共同稳定区域与 $\left(\mathrm{Ni}_{x} \mathrm{Co}_{y} \mathrm{Mn}_{1-x-y}\right)(\mathrm{OH})_{2}$ 共沉淀区 域相交的 $\mathrm{pH}$ 值由 7.5 向左移动至 4.5。这说明随着 温度升高, 金属离子 $\mathrm{Ni}^{2+} 、 \mathrm{Co}^{2+}$ 和 $\mathrm{Mn}^{2+}$ 可以在更低
$\mathrm{pH}$ 值下水解成复合氢氧化物 $\left(\mathrm{Ni}_{x} \mathrm{Co}_{y} \mathrm{Mn}_{1-x-y}\right)(\mathrm{OH})_{2}$ 共沉淀。随着温度升高, $\mathrm{LiNiO}_{2} 、 \mathrm{LiCoO}_{2}$ 和 $\mathrm{LiMnO}_{2}$ 稳定区域向低 $\mathrm{pH}$ 和低电势方向扩大, 如 $\mathrm{LiMnO}_{2}$ 稳 定区域随着温度升高从一小三角形扩大为五边形; $\mathrm{LiNi}_{x} \mathrm{Co}_{y} \mathrm{Mn}_{1-x-y} \mathrm{O}_{2}$ 稳定区域也从 $25^{\circ} \mathrm{C}$ 下不存在变化 为稳定区间 $\mathrm{pH}$ 范围为 9.7 13.0。由此可推断出升高 温度有利于 $\mathrm{LiNi}_{x} \mathrm{Co}_{y} \mathrm{Mn}_{1-x-y} \mathrm{O}_{2}$ 合成。

\section{2 验证实验}

将 $\left(\mathrm{Ni}_{0.5} \mathrm{Co}_{0.2} \mathrm{Mn}_{0.3}\right)(\mathrm{OH})_{2}$ (电池级, 厦门铇业股 份有限公司)、 $\mathrm{LiOH} \cdot \mathrm{H}_{2} \mathrm{O}$ (电池级, 四川天齐锂业有 限公司)和去离子水按比例加入到高压反应釜内, 密封后升温到 $175^{\circ} \mathrm{C}$ 或 $250^{\circ} \mathrm{C}$ 保温 $4 \mathrm{~h}$ 。反应完成后, 将浆料从反应釜中倒出进行液固分离, 所得滤饼 烘干后即得到锂镍钴锰(LNCM)四元前驱体。锂镍 钴锰四元前驱体经过 $500^{\circ} \mathrm{C}$ 低温预处理 $6 \mathrm{~h}, 900^{\circ} \mathrm{C}$ 高温热处理 $10 \mathrm{~h}$, 得到最终产物 $\mathrm{LiNi}_{0.5} \mathrm{Co}_{0.2} \mathrm{Mn}_{0.3} \mathrm{O}_{2}$ 正极材料。

采用日本理学 D/max-rAX 射线衍射仪(XRD, Rigaku 公司, 日本)分析样品的物相组成, 测试条件 为铜靶 $\mathrm{K} a$ 辐射, 管电压 $40 \mathrm{kV}$, 扫描速度 $10 \% \mathrm{~min}$, 步宽 $0.02^{\circ}$, 扫描范围 10 80 。采用日本 JEOL 公司 的 JSM-5600LV 型号的扫描电子显微镜分析材料的 形貌。正极材料、乙炔黑和聚偏氟乙烯(PVDF)按 $8: 1: 1$ 称量混合, 加入一定量的 $\mathrm{N}$-甲基-2-吡络烷 酮(NMP)研磨均匀, 在铝箔上进行涂布, 置于 $130^{\circ} \mathrm{C}$ 真空干燥箱内烘干, 敲成 $14 \mathrm{~mm}$ 的圆形电极片。以 电极片为正极, 金属锂片为负极, 在充满高纯氩气 的手套箱内组装成 CR2016 型纽扣电池, 采用武汉 蓝电测试仪(CT2001A)对组装好的电池进行循环性 能测试。

图 3 是 $250^{\circ} \mathrm{C}$ 下所得锂镍钴锰四元前驱体(图 3(a)) 和热处理后所得 $\mathrm{LiNi}_{0.5} \mathrm{Co}_{0.2} \mathrm{Mn}_{0.3} \mathrm{O}_{2}$ 正极材料(图 3(b)) 的 XRD 图谱, 从图中可以看出, 锂镍钴锰四元前驱 体特征峰的位置与最终产品 $\mathrm{LiNi}_{0.5} \mathrm{Co}_{0.2} \mathrm{Mn}_{0.3} \mathrm{O}_{2}$ 正极 材料基本一致。另外，与标准卡片对比发现，锂镍钴 锰四元前驱体特征峰的位置区别于 $\mathrm{Ni}(\mathrm{OH})_{2}$ 特征峰 (PDF\#14-0117), 而与 $\mathrm{LiNiO}_{2}$ 卡片(PDF\#09-0063)相 近。由此可以推断, $\left(\mathrm{Ni}_{0.5} \mathrm{Co}_{0.2} \mathrm{Mn}_{0.3}\right)(\mathrm{OH})_{2}$ 和 $\mathrm{LiOH} \bullet \mathrm{H}_{2} \mathrm{O}$ 通过水热反应形成了一种与 $\mathrm{LiNi}_{0.5} \mathrm{Co}_{0.2} \mathrm{Mn}_{0.3} \mathrm{O}_{2}$ 正 极材料具有相同结构的材料。将图 3(b)图谱与 $\mathrm{LiNiO}_{2}$ 标准 PDF 卡片对比发现, $\mathrm{LiNi}_{0.5} \mathrm{Co}_{0.2} \mathrm{Mn}_{0.3} \mathrm{O}_{2}$ 正极材 料具有典型的 $\alpha-\mathrm{NaFeO}_{2}$ 层状结构, 空间群为 R-3m。 比较图 3(a)和(b)发现, 锂镍钴锰四元前驱体的峰强 度较弱, 表明其结晶度低, 晶体结构不完善。而经过 


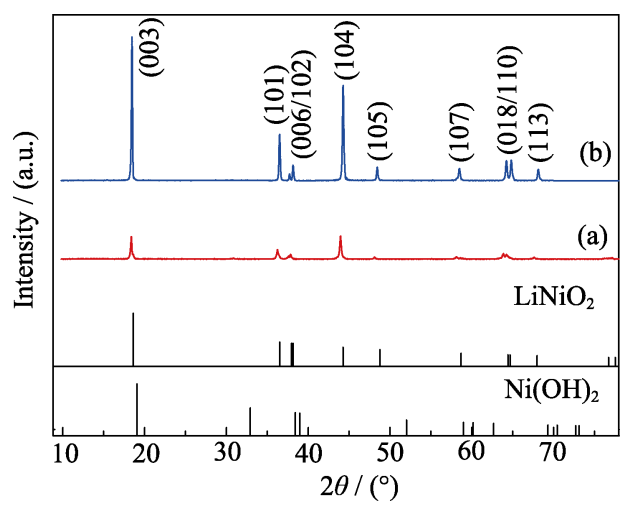

图 3 锂镍钴锰四元前驱体(a) 和 $\mathrm{LiNi}_{0.5} \mathrm{Co}_{0.2} \mathrm{Mn}_{0.3} \mathrm{O}_{2}$ 正极材 料(b)的 XRD 图谱

Fig. 3 XRD patterns of the LNCM oxide precursor (a) and the final product $\mathrm{LiNi}_{0.5} \mathrm{Co}_{0.2} \mathrm{Mn}_{0.3} \mathrm{O}_{2}$ (b)

热处理后, 所得 $\mathrm{LiNi}_{0.5} \mathrm{Co}_{0.2} \mathrm{Mn}_{0.3} \mathrm{O}_{2}$ 正极材料的衍射 峰强度明显增大、衍射峰变得更尖锐，且(006)/(102) 和(018)/(110)两组分裂峰分裂明显, 这说明所得 $\mathrm{LiNi}_{0.5} \mathrm{Co}_{0.2} \mathrm{Mn}_{0.3} \mathrm{O}_{2}$ 正极材料的结晶度良好, 层状结 构完善。采用 Jade 软件由 XRD 图谱中数据计算得 到 $\mathrm{LiNi}_{0.5} \mathrm{Co}_{0.2} \mathrm{Mn}_{0.3} \mathrm{O}_{2}$ 正极材料的晶胞参数, 其中 $a$ 为 $0.28717 \mathrm{~nm}, c$ 为 $1.42467 \mathrm{~nm}, c / a$ 为 4.9611 。实验结 果证实, 通过水溶液法可以合成具有 $\alpha-\mathrm{NaFeO}_{2}$ 层状 结构的锂镍钴锰四元前驱体, 同时表明所绘制 $E-\mathrm{pH}$ 图是可信的。

图 4 是 $175^{\circ} \mathrm{C}$ 和 $250^{\circ} \mathrm{C}$ 下所得 $\mathrm{LiNi}_{0.5} \mathrm{Co}_{0.2} \mathrm{Mn}_{0.3} \mathrm{O}_{2}$ 正极材料首次和第 50 次充/放电曲线。从图中可知, 在电压范围为 $3.0 \sim 4.3 \mathrm{~V}$ 下, $175^{\circ} \mathrm{C}$ 和 $250^{\circ} \mathrm{C}$ 下所得 $\mathrm{LiNi}_{0.5} \mathrm{Co}_{0.2} \mathrm{Mn}_{0.3} \mathrm{O}_{2}$ 正极材料的 $0.1 \mathrm{C}$ 首次充/放电比 容量分别为 186.0/163.4 mAh/g、207.3/169.8 mAh/g; $0.5 \mathrm{C}$ 首次放电比容量分别为 $151.2 \mathrm{mAh} / \mathrm{g}$ 和 $154.3 \mathrm{mAh} / \mathrm{g}$ 。图 5 为 $175{ }^{\circ} \mathrm{C}$ 和 $250{ }^{\circ} \mathrm{C}$ 下所得 $\mathrm{LiNi}_{0.5} \mathrm{Co}_{0.2} \mathrm{Mn}_{0.3} \mathrm{O}_{2}$ 正极材料在 $0.5 \mathrm{C}$ 下的放电循环 曲线和容量保持率曲线。在 $0.5 C$ 、电压范围分别为 3.0 4.3 V下, $175^{\circ} \mathrm{C}$ 和 $250^{\circ} \mathrm{C}$ 下所得 $\mathrm{LiNi}_{0.5} \mathrm{Co}_{0.2} \mathrm{Mn}_{0.3} \mathrm{O}_{2}$ 正极材料循环 50 圈后, 放电比容量分别为 141.5 和 $151.6 \mathrm{mAh} / \mathrm{g}$, 容量保持率分别达 93.6\%和 98.2\%。

从图 5 可以看出, 在前 15 次循环过程中, $\mathrm{LiNi}_{0.5} \mathrm{Co}_{0.2} \mathrm{Mn}_{0.3} \mathrm{O}_{2}$ 正极材料具有较高的容量保持率; 循环 15 圈之后, $250^{\circ} \mathrm{C}$ 下所得 $\mathrm{LiNi}_{0.5} \mathrm{Co}_{0.2} \mathrm{Mn}_{0.3} \mathrm{O}_{2}$ 正 极材料的容量保持率曲线依然较平稳且接近 $100 \%$, 而 $175^{\circ} \mathrm{C}$ 下所得 $\mathrm{LiNi}_{0.5} \mathrm{Co}_{0.2} \mathrm{Mn}_{0.3} \mathrm{O}_{2}$ 正极材料的容量 保持率曲线则逐渐下降, 容量保持率降低。这说明 $250^{\circ} \mathrm{C}$ 下所得 $\mathrm{LiNi}_{0.5} \mathrm{Co}_{0.2} \mathrm{Mn}_{0.3} \mathrm{O}_{2}$ 正极材料具有更高 的放电比容量和更好的循环稳定性, 与 $\mathrm{Li}-\mathrm{Ni}$ Co-Mn- $\mathrm{H}_{2} \mathrm{O}$ 系优势区域图显示的结果一致, 因为温 度升高, $\mathrm{LiNi}_{x} \mathrm{Co}_{y} \mathrm{Mn}_{1-x-y} \mathrm{O}_{2}$ 的稳定区域扩大, 制备

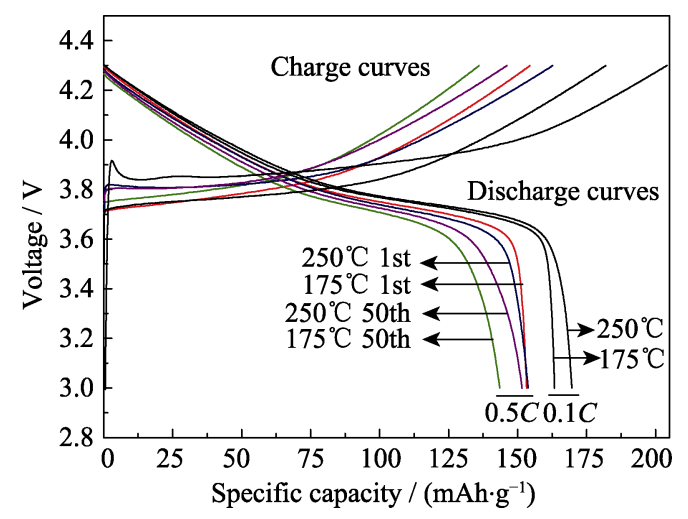

图 $4175^{\circ} \mathrm{C}$ 和 $250^{\circ} \mathrm{C}$ 下所得 $\mathrm{LiNi}_{0.5} \mathrm{Co}_{0.2} \mathrm{Mn}_{0.3} \mathrm{O}_{2}$ 正极材料的 充/放电曲线

Fig. 4 Charge/discharge curves of the $\mathrm{LiNi}_{0.5} \mathrm{Co}_{0.2} \mathrm{Mn}_{0.3} \mathrm{O}_{2}$ cathode materials synthesized at $175^{\circ} \mathrm{C}$ and $250^{\circ} \mathrm{C}$

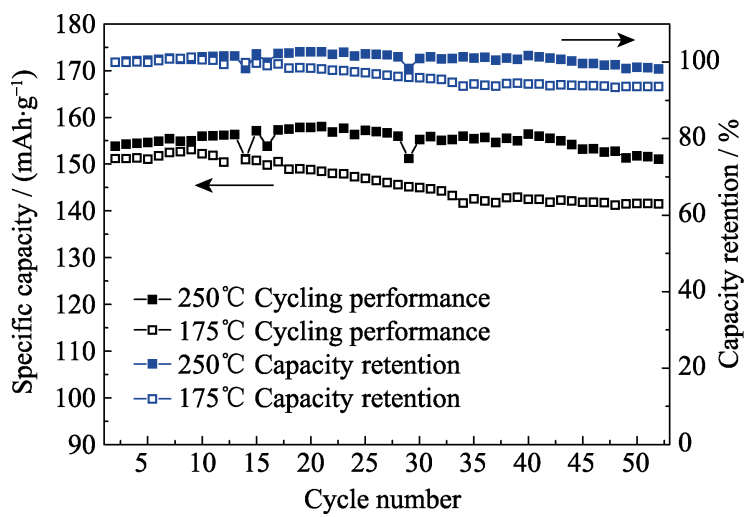

图 $5175^{\circ} \mathrm{C}$ 和 $250^{\circ} \mathrm{C}$ 下所得 $\mathrm{LiNi}_{0.5} \mathrm{Co}_{0.2} \mathrm{Mn}_{0.3} \mathrm{O}_{2}$ 正极材料在 $0.5 C$ 下的放电循环曲线和容量保持率曲线

Fig. 5 Discharge cycling performance and capacity retention rate of the $\mathrm{LiNi}_{0.5} \mathrm{Co}_{0.2} \mathrm{Mn}_{0.3} \mathrm{O}_{2}$ cathode materials synthesized at $175^{\circ} \mathrm{C}$ and $250^{\circ} \mathrm{C}$

$\mathrm{LiNi}_{x} \mathrm{Co}_{y} \mathrm{Mn}_{1-x-y} \mathrm{O}_{2}$ 时, 其稳定区域周围杂相出现的 可能性降低, 有利于合成纯相结构 $\mathrm{LiNi}_{x} \mathrm{Co}_{y} \mathrm{Mn}_{1-x-y} \mathrm{O}_{2}$ 材料, 实验结果证实了所绘制的 $E-\mathrm{pH}$ 图的可靠性。

\section{3 结论}

绘制了 $25^{\circ} \mathrm{C}$ 和 $200^{\circ} \mathrm{C}$ 、溶液中离子活度为 1.00 下 Li-Ni-Co-Mn- $\mathrm{H}_{2} \mathrm{O}$ 系的 E-pH 图。热力学预测表 明: 水溶液中存在 $\mathrm{LiNi}_{x} \mathrm{Co}_{y} \mathrm{Mn}_{1-x-y} \mathrm{O}_{2}$ 的稳定区域; 随着温度升高, $\mathrm{LiNi}_{x} \mathrm{Co}_{y} \mathrm{Mn}_{1-x-y} \mathrm{O}_{2}$ 稳定区域向低 $\mathrm{pH}$ 和低电位方向扩大。因此, 在水溶液中, 控制适宜的 条件合成 $\mathrm{LiNi}_{x} \mathrm{Co}_{y} \mathrm{Mn}_{1-x-y} \mathrm{O}_{2}$ 材料是可能的, 且提高 温度有利于材料的合成。通过水溶液中的验证实验, 分别在 $175^{\circ} \mathrm{C}$ 和 $250^{\circ} \mathrm{C}$ 下合成了具有 $\alpha-\mathrm{NaFeO}_{2}$ 层状 结构的 $\mathrm{LiNi}_{0.5} \mathrm{Co}_{0.2} \mathrm{Mn}_{0.3} \mathrm{O}_{2}$ 正极材料。相比之下, $250^{\circ} \mathrm{C}$ 下得到的 $\mathrm{LiNi}_{0.5} \mathrm{Co}_{0.2} \mathrm{Mn}_{0.3} \mathrm{O}_{2}$ 正极材料, 放电 比容量更高、循环性能更良好验证了热力学预测的 可靠性。 


\section{参考文献:}

[1] LI L, LI Y J, XU C, et al. Eh-pH diagrams from $333.15 \mathrm{~K}$ to $453.15 \mathrm{~K}$ for lithium titanium composite oxides and their synthesis in aquous solution. Hydrometallugy, 2014, 142: 131-136.

[2] PORTHAULT H, LE CRAS F, BADOUR-HADJEAN R, et al. One step synthesis of lamellar R-3m $\mathrm{LiCoO}_{2}$ thin films by an electrochemical-hydrothermal method. Electrochimica Acta, 2011, 56(22): 7580-7585.

[3] GUO C H, ZHAO Z W, HUO G S. Thermodynamics analysis on Li-Ni- $\mathrm{H}_{2} \mathrm{O}$ system. Chinese Journal of Power and Sources, 2005, 29(6): 376-379.

[4] WEN S M, ZHAO Z W, HUO G S. Thermodynamic analysis and potential-pH diagrams of $\mathrm{Li}-\mathrm{Co}-\mathrm{H}_{2} \mathrm{O}$ system. Chinese Journal of Power and Sources, 2005, 29(7): 423-426.

[5] ZHAO Z W, HUO G. Thermodynamic analysis on Li-Mn- $\mathrm{H}_{2} \mathrm{O}$ system. The Chinese Journal of Nonferrous Metals, 2004, 14(11): 1926-1933.

[6] QIU W S, LI Y J, ZHAO Z W, et al. system at $298.15 \mathrm{~K}$ and synthesis of $\mathrm{Li}_{4} \mathrm{Ti}_{5} \mathrm{O}_{12}$. The Chinese Journal of Nonferrous Metals, 2010, 20(11): 2260-2268.

[7] FERGUS J W. Recent developments in cathode materials for lithium ion batteries. J. Power Sources, 2010, 195(4): 939-954.

[8] NOH H J, YOUN S, YOON C S, et al. Comparison of the structural and electrochemical properties of layered $\mathrm{Li}\left[\mathrm{Ni}_{x} \mathrm{Co}_{y} \mathrm{Mn}_{z}\right] \mathrm{O}_{2}$ $(x=1 / 3,0.5,0.6,0.7,0.8$ and 0.85$)$ cathode material for lithium-ion batteries. J. Power Sources, 2013, 233: 121-130.
[9] OHZUKU T, MAKIMURAAK Y. Layered lithium insertion material of $\mathrm{LiCo}_{1 / 3} \mathrm{Ni}_{1 / 3} \mathrm{Mn}_{1 / 3} \mathrm{O}_{2}$ for lithium-ion batteries. Chem. Letters, 2001(7): 642-643.

[10] LEE M H, KANG Y J, MYUNG S T, et al. Synthetic optimization of $\mathrm{Li}\left[\mathrm{Ni}_{1 / 3} \mathrm{Co}_{1 / 3} \mathrm{Mn}_{1 / 3}\right] \mathrm{O}_{2}$ via co-precipitation. Electrochimica Acta, 2004, 50(4): 939-948.

[11] XIA $\mathrm{H}$, WANG $\mathrm{H}$, XIAO $\mathrm{W}$, et al. Properties of $\mathrm{LiNi}_{1 / 3} \mathrm{Co}_{1 / 3} \mathrm{Mn}_{1 / 3} \mathrm{O}_{2}$ cathode material synthesized by a modified Pechini method for high-power lithium-ion batteries. J. Alloys. Compd, 2009, 480(2): 696-701.

[12] HU X S, LIU $X$ Q. Synthesis of cathode material $\mathrm{LiNi}_{1 / 3} \mathrm{Co}_{1 / 3} \mathrm{Mn}_{1 / 3} \mathrm{O}_{2}$ and its electrochemical performances. Chinese Journal of Power and Sources. 2006, 30(3): 183-186.

[13] LU H Q, ZHOU H T, SVENSSON A M, et al. High capacity $\mathrm{Li}\left[\mathrm{Ni}_{0.8} \mathrm{Co}_{0.1} \mathrm{Mn}_{0.1}\right] \mathrm{O}_{2}$ synthesized by Sol-Gel and co-precipitation methods as cathode materials for lithium-ion batteries. Solid State Ionics, 2013, 249-250: 105-111.

[14] LI D C, MUTA T, ZHANG L Q, et al. Effect of synthesis method on the electrochemical performance of $\mathrm{LiNi}_{1 / 3} \mathrm{Mn}_{1 / 3} \mathrm{Co}_{1 / 3} \mathrm{O}_{2} . J$. Power Sources, 2004, 132(1/2): 150-155.

[15] MYUNG S T, LEE M H, KOMABA S, et al. Hydrothermal synthesis of layered $\mathrm{Li}\left[\mathrm{Ni}_{1 / 3} \mathrm{Co}_{1 / 3} \mathrm{Mn}_{1 / 3}\right] \mathrm{O}_{2}$ as positive electrode material for lithium secondary battery. Electrochimica Acta, 2005, 50(24): 4800-4806.

[16] SPEIGHT J. Lange’s Chemistry Handbook. McGraw-Hill Professional, 2004: 1246-1259.

[17] CONNICK R E, POWELL R E. The entropy of aqueous oxy anions. J. Chem. Phys. 1953, 21(12): 2206-2207. 